\title{
Verification of a New Quantum Simulation Approach through Its Application to Heisenberg-Like Models
}

\author{
ZHAOSEN LIU ${ }^{a, *}$ AND HOU IAN ${ }^{b}$ \\ ${ }^{a}$ Department of Applied Physics, Nanjing University of Information Science and Technology, Nanjing 210044, China \\ ${ }^{b}$ Institute of Applied Physics and Materials Engineering, FST, University of Macau, China
}

(Received March 30, 2016; in final form May 17, 2016)

\begin{abstract}
A quantum simulation approach facilitated by the self-consistent algorithm was applied in the present work to ferromagnetic and antiferromagnetic three-dimensional Heisenberg lattices consisting of $S=1$ spins. Consequently, the calculated spontaneous magnetizations for the two sorts of lattices are precisely consistent with mean-field theory in the whole temperature range. Especially, the numerical results, such as magnetizations, total energies and total free energies per mole of spins, show no size effects. Thus, the physical properties of a huge bulk magnet can be estimated by performing simulation for a very tiny sample, so that the computational time can be greatly saved.
\end{abstract}

DOI: 10.12693/APhysPolA.129.1210

PACS/topics: $75.40 . \mathrm{Mg}, 75.10 . \mathrm{Jm}$

\section{Introduction}

Even nowadays, the Heisenberg model is still a very hot research topic which are currently intensively studied both theoretically with quantum physics [1-5] and numerically mainly with Monte Carlo (MC) method [6-10]. However, the MC method is based on classical physics, since all spins in the studied systems are treated by the method as classical vectors with fixed lengths, though they are allowed to rotate spatially in the computing process. On the other hand, the mean-field theory is now still very popular both in classical and quantum statistical model description, and various new methods have been proposed to develop the theory and improve the approximation [11-13]. Recently, Dudek et al. combined the mean-field approximation with the Monte Carlo scheme to propose a new simulation method, as a result, their calculated magnetization with this model for the twodimensional Ising model is well consistent with mean-field theory [14].

To cope with the problem met by the classical methods, we have developed a new quantum simulation method based on mean-field theory by using a self-consistent algorithm, so it is referred to as the SCA approach, and have successfully applied it to nanosystems consisting of rare-earth elements $[15,16]$ and $3 d$ transition metals [17-19]. The SCA approach was assumed to be based on the principle of the lowest free energy [15]. That is, in a computing process, the orientations and magnitudes of all magnetic moments in the considered system are adjusted simultaneously by the local effective magnetic fields to minimize the total free energy of the whole system, so that the code can quickly converge to correct equilibrium states at all temperatures

\footnotetext{
*corresponding author; e-mail: liuzhsnj@yahoo.com
}

spontaneously. The above hypothesis has been proved by our recent simulations performed for a ferromagnetic (FM) nanowire [18].

So far, our calculated results are all reasonable, especially, our simulated magnetic structure using the SCA approach for a $\mathrm{DyNi}_{2} \mathrm{~B}_{2} \mathrm{C}$ nanoball, assumed to be cut out of a body-centered tetragonal crystallite, were in good agreement with that observed in the bulk sample: below the transition temperature, the magnetic moments on an $a b$-plane inside the core aligned ferromagnetically in the [110] direction, two adjacent $a b$ layers ordered antiferromagnetically, and the calculated transition temperature was very close to that of the bulk sample [16]. In addition, our recent simulations performed for an antiferromagnetic nanoparticle using the SCA approach has generated the same results as those calculated with a new quantum Monte Carlo method we proposed, so that the two quantum simulation approaches were verified by each other [19].

However, to prove the correctness of this new simulation method, it is still necessary to make comparison directly with quantum theory. For the purpose, this work has been done. Here, the SCA approach was applied to three-dimensional (3D) Heisenberg-like models, and the simulated results were compared with those obtained by directly solving the system Hamiltonians. Consequently, the calculated magnetizations in the two ways are exactly identical, verifying the correctness of the new simulation approach theoretically. Moreover, the simulated results show no size effects. Thus, the physical properties of a huge bulk magnet can be obtained by performing simulations for a very tiny sample, so that the computational speed can be greatly accelerated.

\section{Quantum simulation model}

In the present work, the magnetic systems are assumed to be composed of $S=1$ spins. When no external 
magnetic field is exerted but uniaxial anisotropy interaction is present, the Heisenberg model can be described by

$$
\mathcal{H}=-\frac{1}{2} \sum_{i, j \neq i} \mathcal{J}_{i j} \boldsymbol{S}_{i} \cdot \boldsymbol{S}_{j}-K_{V} \sum_{i} S_{z}^{2},
$$

where $\mathcal{J}_{i j}$ and $K_{V}$ denotes the strengths of the Heisenberg exchange interaction among the neighboring spins, and the magnetic uniaxial anisotropy assumed to be in the $z$-direction.

For simplicity, we only consider the exchange interaction between the nearest spins, and further assume that this interaction is uniform in the whole magnet, i.e., $\mathcal{J}_{i j}=\mathcal{J}$. In our model, the spins appearing in Eq. (1) are quantum operators instead of the classical vectors. Since $S=1$, the matrices of the three spin components are given by

$$
\begin{gathered}
S_{x}=\frac{1}{2}\left(\begin{array}{ccc}
0 & \sqrt{2} & 0 \\
\sqrt{2} & 0 & \sqrt{2} \\
0 & \sqrt{2} & 0
\end{array}\right), S_{y}=\frac{1}{2 \mathrm{i}}\left(\begin{array}{ccc}
0 & \sqrt{2} & 0 \\
-\sqrt{2} & 0 & -\sqrt{2} \\
0 & \sqrt{2} & 0
\end{array}\right), \\
S_{z}=\left(\begin{array}{ccc}
1 & 0 & 0 \\
0 & 0 & 0 \\
0 & 0 & -1
\end{array}\right)
\end{gathered}
$$

respectively, and the thermal average of any physical quantity $A$ at temperature $T$ are evaluated with

$$
\langle A\rangle=\frac{\sum_{n}\left\langle\varphi_{n}\left|\hat{A} \exp \left(-\varepsilon_{n} / k_{\mathrm{B}} T\right)\right| \varphi_{n}\right\rangle}{\sum_{n} \exp \left(-\varepsilon_{n} / k_{\mathrm{B}} T\right)},
$$

where $\varepsilon_{n}$ and $\varphi_{n}$ are the eigenenergy and eigenfunction of the considered spin Hamiltonian.

A simple antiferromagnetic system can be decomposed into two sublattices which order in the opposite directions in the magnetic phase. Thus, the spins in the same sublattice are coupled ferromagnetically, but those belonging to different sublattices are coupled antiferromagnetically. Once again, if we only consider the uniform nearest neighboring exchange interaction, the Hamiltonian for each kind of the system has the similar form, but now $\mathcal{J}<0$, and in the computing process we must know clearly what a spin is considered and what are the neighboring spins.

In our theoretical computations, the above Hamiltonian was firstly diagonalized to obtain the eigenvalues and eigenfunctions of the considered spin, then they were inserted into quantum formulae to calculate the $\left\langle S_{x}\right\rangle$, $\left\langle S_{y}\right\rangle$ and $\left\langle S_{z}\right\rangle$ of the spins in a self-consistent manner.

To make use of the SCA approach for spin lattices, mean-field theory must be employed. Thus, the Hamiltonian is decomposed into $N$ non-linear equations which are still coupled together, provided that the lattice contains $N$ spins. Then in the simulation, these non-linear equations are solved numerically and the $N$ magnetic moments are evaluated self-consistently. The SCA simulations are usually started far above the transition temperatures from a spin configuration with all spins randomly orientated, then carried out down to very low temperatures with a reducing step $\Delta T(<0)$, and periodical boundary conditions are applied. During simulations, once the difference $\left|\left\langle S_{i}^{\prime}\right\rangle-\left\langle S_{i}\right\rangle\right|$ between the two successive iterations for every spin is less than a very small given value $\tau_{0}$, convergency is considered to be reached.

\section{Numerical results in comparison with mean-field theory}

To compare with the theory, we performed simulations for both ferromagnetic (FM) and antiferromagnetic (AFM) 3D magnetic lattices consisting of $S=1$ spins by means of the SCA approaches using $\mathcal{J}= \pm 1 \mathrm{~K}$ and $K_{V}=1 \mathrm{~K}$, respectively. Here, the two parameters are scaled with the Boltzmann constant. Moreover, since $\mathcal{J}$ is assigned to the unit, all other parameters and quantities, including temperature, energy and free energy are scaled with $\mathcal{J}$ as well, so that our calculated results have more general meaning.

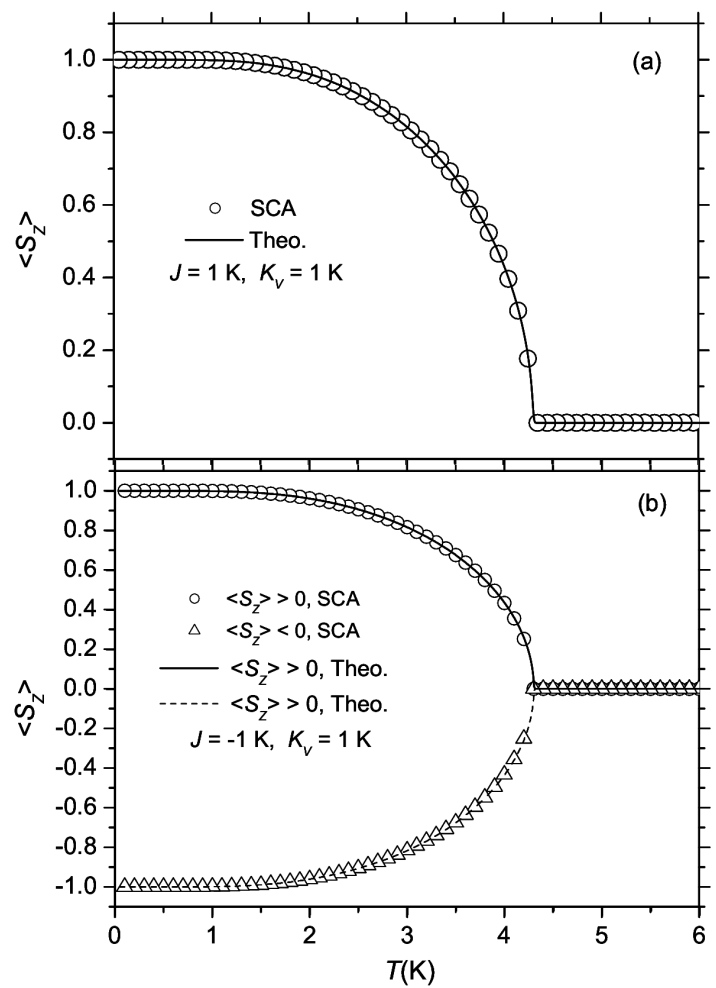

Fig. 1. Spontaneous magnetizations calculated with the SCA approach for the (a) FM, and (b) AFM, 3D Heisenberg models on cubic lattice in comparison with mean-field theory.

The spontaneous $\left\langle S_{z}\right\rangle$ curves calculated with the set of parameters for the two sorts of lattices are depicted in Fig. 1, other two components, $\left\langle S_{x}\right\rangle$ and $\left\langle S_{y}\right\rangle$, vanish due to the strong uniaxial anisotropy along the $z$-axis. In the both cases, our numerical results are precisely identical with those obtained by directly diagonalizing the Hamiltonian matrices, so the transition temperatures detected from the curves, being about $4.32 \mathrm{~K}$, are exactly equal to the theoretical value. 

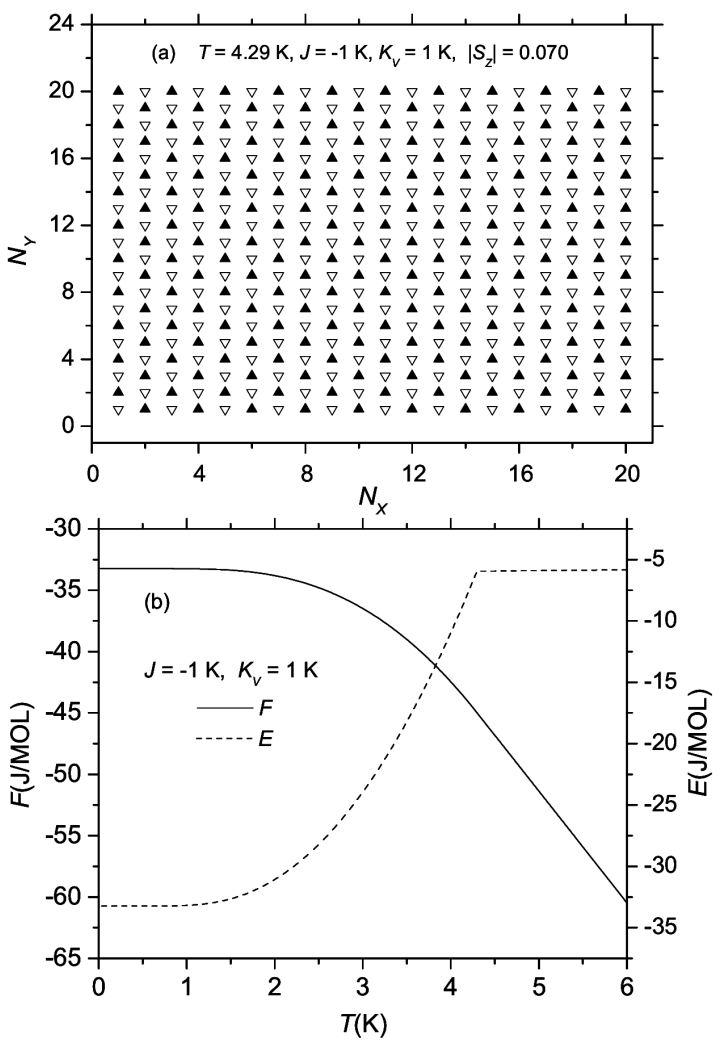

Fig. 2. (a) Magnetic configuration on the center (001) cross-section obtained at $T=4.29 \mathrm{~K}$ for the AFM 3D Heisenberg model on cubic lattice, (b) free energy and energy per mole of spins calculated for both FM and AFM 3D Heisenberg models on cubic lattice, in the absence of external magnetic field by means of the SCA method. The solid and hollow triangles denote the positive and negative spins in the $z$-direction, respectively.

Our simulations with the SCA approach have generated almost perfect ferromagnetic and antiferromagnetic magnetic configurations in the magnetic phases even immediately below the transition temperatures as shown in Fig. 2a, for instance, where the spins on the $z=10 a$ cross-section obtained at $T=4.29 \mathrm{~K}$ for $20 \times 20 \times 20 \mathrm{AFM}$ lattice are projected onto the (001) plane. In the case of ferromagnet, all spins order ferromagnetically below $T_{\mathrm{C}}$, so there is no need to plot such simple spin structure here.

For these canonical systems, the total free energy $F$ and total energy $E$ can be calculated with $F=$ $-k_{\mathrm{B}} T \log Z_{N}$ and $E=-\frac{\partial}{\partial \beta} \log Z_{N}$ respectively, where $\beta=1 /\left(k_{\mathrm{B}} T\right)$ and $Z_{N}$ is the partition function of the whole system. Figure $2 \mathrm{~b}$ displays the $F$ and $E$ curves obtained with the SCA method. Astonishingly, both the total free energies and total energies calculated by means of the SCA approach for the FM and AFM lattices are precisely identical with each other. The sudden change in the $E$ curve exactly at $T_{N}$ is a sign of phase transition.

We have repeated simulations for FM and AFM Heisenberg lattices with different sizes, of course using the periodic conditions and the same set of parameters, and always obtained the same magnetizations, total energies and total free energies per mole of spins as displayed in the above two figures.

\section{Computational efficiency and accuracy}

To study the computational efficiency, we carried out simulations for a 3D AFM Heisenberg-like lattice consisting of $10 \times 10 \times 10$ spins by assigning $\Delta T=-0.05 \mathrm{~K}$ and $\tau_{0}=10^{-7}$, the iterations required to reach convergency at different temperatures are displayed in Fig. 3a. The loop number varies with decreasing temperature, a sharp peak appears around $T_{N} \approx 4.32 \mathrm{~K}$, meaning much more iterations are needed to reach equilibrium state near phase transition, then attenuates rapidly as temperature drops. Very strikingly, it only took less than 10 loops to converge below $2.65 \mathrm{~K}$, and about $35.1 \mathrm{~s}$ to complete the whole simulation.

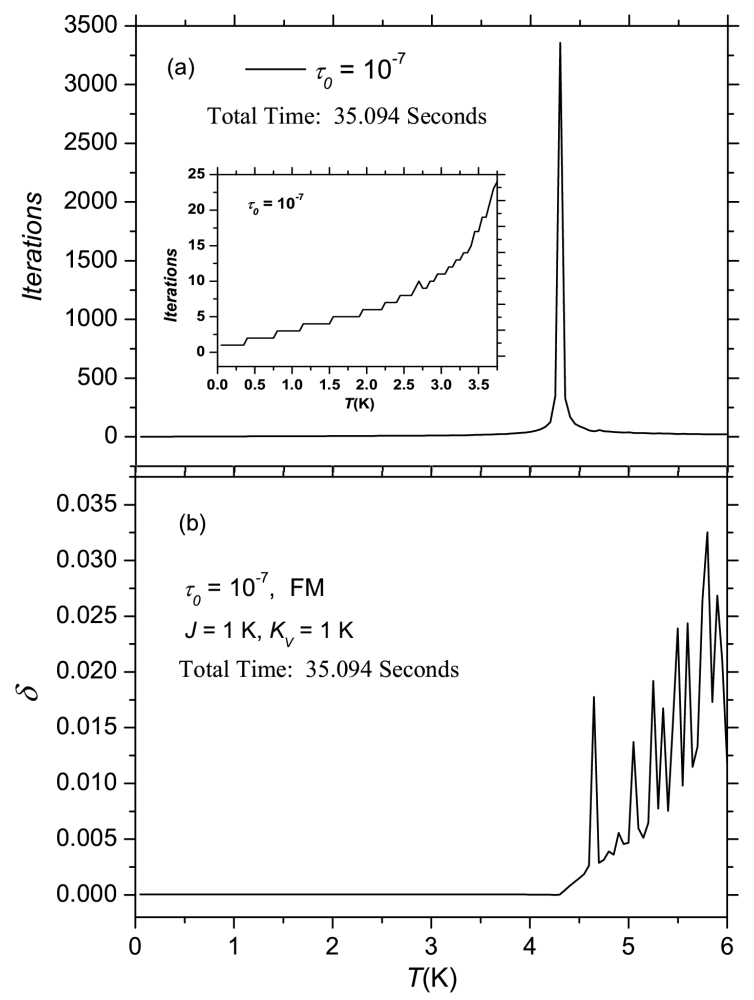

Fig. 3. (a) Iterations required to reach equilibrium at different temperatures, and (b) variances estimated after convergency, as the SCA approaches was applied to the $10 \times 10 \times 10 \mathrm{FM}$ Heisenberg lattice.

On the other hand, to estimate the computational accuracy, we define the variance as:

$$
\delta=\frac{1}{N \overline{\left\langle S_{z}\right\rangle}} \sqrt{\sum_{i}\left(\left\langle S_{i z}\right\rangle-\overline{\left\langle S_{z}\right\rangle}\right)^{2}},
$$

where $N$ is the total number of the spins in the lattice. Figure $3 \mathrm{~b}$ displays $\delta$ curve obtained in the SCA simulation for the $10 \times 10 \times 10 \mathrm{FM}$ lattice. The relative larger $\delta$ values above $T_{\mathrm{C}}(\approx 4.32 \mathrm{~K})$ reflect violent spin fluctuations. The $\delta$ value drops sharply as $T<T_{\mathrm{C}}$. For 
instance, $\delta \sim 3 \times 10^{-3}$ below $T=4.2 \mathrm{~K}$. This small value reflects very good computational accuracy achieved by the simulation approach.

\section{Conclusions and discussion}

To check our results presented here, we repeated simulations later for the $20 \times 20 \times 20$ lattices by increasing iteration number up to 5000, however found no obvious differences. We also performed simulations for the FM and AFM Heisenberg lattices of size $4 \times 4 \times 4$ using the same set of parameters and periodical boundary conditions, consequently obtained exactly same results as those shown in Figs. 1 and Fig. 2b. Of course, the calculated magnetic structures for the two small lattices are perfectly ferromagnetic or antiferromagnetic. Especially, as $\tau_{0}=10^{-7}$ and $\Delta T=-0.02 \mathrm{~K}$, the simulation for the tiny AFM lattice only took about $8.94 \mathrm{~s}$ to complete. Therefore, the properties of a huge magnet can be determined directly by doing simulation for a very tiny sample with the SCA approach, so that the computational speed can be greatly accelerated, at least in the present cases.

By reducing $K_{V}$ from $1 \mathrm{~K}$ to very small values for the FM and AFM Heisenberg lattices, we found in simulations that the $\left\langle S_{z}\right\rangle$ curves obtained with the numerical and theoretical methods were always identical, no nonzero $\left\langle S_{x}\right\rangle$ and $\left\langle S_{y}\right\rangle$ were visible, except for the case when $K_{V}$ is extremely weak, for example as $K_{V}=0.01 \mathrm{~K}$, where both $\left\langle S_{x}\right\rangle$ and $\left\langle S_{y}\right\rangle$ were detectable in an order of $2 \times 10^{-5}$ around $T_{M}$, thus negligible.

In conclusion, we have applied the SCA approach to the FM and AFM Heisenberg-like models, and the calculated magnetizations are identical with those obtained by directly diagonalizing the Hamiltonians. Since the periodical boundary condition was used in the frame of quantum physics, in the FM case, all spins in the lattice are same; whereas in the AFM case, all spins belonging to the same sublattice are identical. Therefore, the whole FM lattice can be represented by just one spin, and the two oppositely oriented sublattices of the AFM lattice may be described by just two antiferromagnetically coupled spins. The agreements between our numerical and theoretical results verify the correctness and applicability of the new simulation approach once again.

\section{Acknowledgments}

Z.S. Liu is supported by National Natural Science Foundation of China under grant No. 11274177. H. Ian is supported by the FDCT of Macau under grant 013/2013/A1, University of Macau under grants MRG022/IH/2013/FST and MYRG2014-00052FST, and National Natural Science Foundation of China under Grant No. 11404415.

\section{References}

[1] G.S. Hu, R.G. Zhu, Physica B 459, 16 (2015).

[2] A.Y. Hu, A.J. Zhang, J. Magn. Magn. Mater. 399, $22(2016)$.

[3] G. Mert, J. Magn. Magn. Mater. 394, 126 (2015).

[4] G. Mert, J. Magn. Magn. Mater. 363, 224 (2014).

[5] S. Pramanik, M. Moussa, M. Faizal, A.F. Ali, Ann. Phys. 362, 24 (2015).

[6] Y.L. Zhou, B.Q. Ou, W. Wu, Phys. Lett. A 379, 2569 (2015).

[7] P.S. Rakić, S.M. Radošević, P.M. Mali, L.M. Stričević, T.D. Petrić, Physica A 441, 69 (2016).

[8] S. Naji, A. Benyoussef, A.E. Kenz, Physica A 391, 3885 (2012).

[9] W. Wang, D. Lv, F. Zhang, J.L. Bi, J.N. Chen, J. Magn. Magn. Mater. 385, 16 (2015).

[10] K. Binder, D.W. Heermann, Monte Carlo Simulation in Statistical Physics. An Introduction, 4. enl. ed., Springer-Verlag, Berlin, Heidelberg, New York, 2002.

[11] M. Opper, D. Saad, Advanced Mean Field Methods, Theory and Practice, MIT Press, Cambridge 2001.

[12] P. Carbonetto, N. de Freitas, in: Conditional Mean Field in Advances in Neural Information Processing Systems 19, Eds. B. Scholkope, J. Platt, T. Hofman, Massachusetts Institute of Technology, Cambridge 2007, p. 201.

[13] R.R. Netz, A.N. Berker, Phys. Rev. Lett. 66, 377 (1991).

[14] M.R. Dudek, J.N. Grima, R. Cauchi, C. Zerafa, R. Gatt, B. Zapotoczny, J. Stat. Phys. 154, 1508 (2014).

[15] Z.-S. Liu, V. Sechovský, M. Diviš, J. Phys. Condens. Matter 23, 016002 (2011).

[16] Z.-S. Liu, V. Sechovský, M. Diviš, Phys. Status Solidi B 249, 202 (2012).

[17] Z.-S. Liu, V. Sechovský, M. Diviš, Physica E 44, 826 (2012).

[18] Z.-S. Liu, V. Sechovský, M. Diviš, Physica E 59, 27 (2014).

[19] Z.-S. Liu, V. Sechovský, M. Diviš, Physica E 62, 123 (2014). 